\title{
Flux Position Estimation using Current Derivatives for the Sensorless Control of AC Machines
}

\author{
James Borg Bartolo \\ Cyril Spiteri Staines \\ Cedric Caruana \\ Department of Industrial Electric Power Conversion, \\ University of Malta, \\ Tal-Qroqq Malta
}

\begin{abstract}
This paper considers the general principles of saliency tracking in AC machines. Special attention is given to pulse type injection for the extraction of a rotor or flux position signal. The saliency is tracked by measuring the current derivative resulting from voltage 'test' vector application. Results show tracking of 'saturation' saliency on a standard induction motor to be used for sensorless control of ac machines in the low and zero speed region.
\end{abstract}

Keywords - Sensorless Control, Saturation Saliency, Current Derivative.

\section{INTRODUCTION}

The induction motor is one of the most popular electrical machines used in industrial applications. For high performance speed and/or torque control, accurate rotor position information is required. Position feedback is usually obtained by encoders which however present a financial overhead in the drive apart from being very fragile when compared to the actual induction machine. Due to these shortcomings, research into sensorless control investigates ways and means to bypass the encoder and get the relevant speed and position signals. Model based sensorless control algorithms estimate the flux position from the terminal quantities of the machine. Such techniques are however parameter dependant.

Signal Injection based techniques track machine saliencies, making them parameter independant. This paper reviews the main types of saliencies found in an induction machine. Results for one non model based method, using pulse test vector injection, are presented. This paper also discusses an inexpensive current derivative sensor that is used for the extraction of position information.

\section{SALIENCIES PRESENT IN INDUCTION MACHINES}

There are mainly two types of saliencies, which might significantly modulate the machine's inductances. The first type of saliency considered is related to the saturation of the stator and rotor teeth under the influence of the main flux setup by the stator and linking the rotor, and leakage flux, as shown in Fig.1. The leakage flux can be defined as the flux setup by either the stator windings or rotor bars which does not cross the air gap. Leakage flux tends to saturate the neighbouring stator and rotor, teeth or bridges, (if present) respectively.

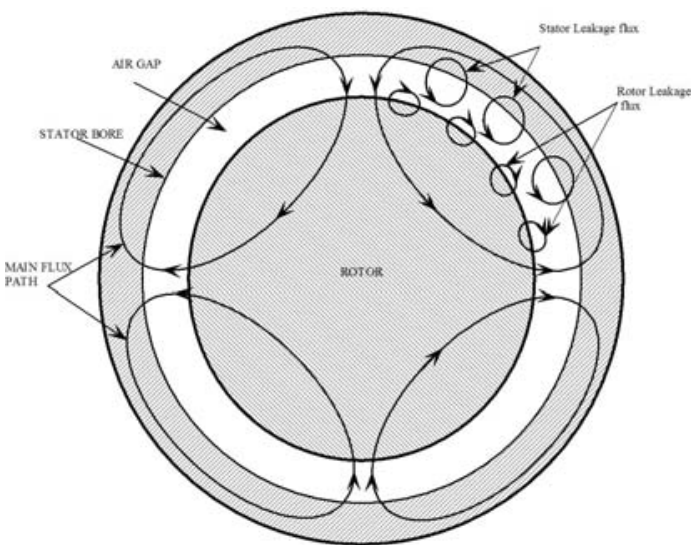

Fig.1. Simplified diagram showing the resultant Flux distribution of a 2 pole pair induction machine.

The other main type of saliency present in a induction machine is rotor slotting effect [3], which allows extraction of rotor position. This is generally a function of the rotor geometry. Just like the saturation effects the latter are generally inherent to most machines. In certain cases, their effects are shadowed by the stronger main flux saturation. To counteract this, in some cases where rotor position is required, the extracted signal is compensated for using complex algorithms.

\section{A. Saturation Saliency}

The type of saliency of particular interest to this study is saturation saliency. It allows for the estimation of the flux position within the machine in sensorless torque control drives. An explanation of the effects of the main flux is considered showing how the resulting permeability modulation varies at twice the rate of the spatial flux distribution within the machine.

The flux generated by the stator windings crossing the air gap and coupling with the rotor bars is defined as the Main flux. The MMF setup per phase by the stator windings along the perimeter of the machine's bore is given by:

$$
\operatorname{MMF} \quad(\theta, t)=N(\theta) \cdot K_{w f} \cdot I(t)
$$

where: $N(\theta)$ is the number of windings as a function mechanical angle; $\mathrm{K}_{\mathrm{wf}}$ is the winding factor allowing 
for spatial distribution of windings and $I(t)$ is the current passing through the phase winding.

It can therefore be shown that a system of three phase currents having frequency $\omega_{\mathrm{e}}$ passing through a number of distributed windings spatially displaced by $2 \pi / 3$ radians will produce a resultant rotating MMF vector given by:

$$
\underline{M}_{\alpha \beta}=M e^{j \omega_{e} t}
$$

where: $\underline{M}_{\alpha \beta}$ is the resultant MMF tensor which rotates along the machine bore coupling the rotor to the stator at the same frequency as the currents supplied to the stator; $M$ is the magnitude of the MMF waveform per phase and $e^{j \omega_{e} t}$ is the rotational operator.

Considering the per phase MMF fundamental to be perfectly sinusoidal and the fact that the permeability $\mu$ of any material is given by:

$$
\mu=\frac{d B}{d H} \alpha \frac{d \phi}{d I}
$$

where $d B / d H$ is the change in magnetic flux density over the magnetic field intensity defined in machine theory as the MMF. Materials such as Silicon Steel saturate, the value of $B$ at which this occurs is denoted by $B_{s}$ and is called the saturation flux density. As the machine is loaded more current will flow through its windings thus increasing the magnetic field intensity and therefore the flux density in the pole neighbourhood. As $\mathrm{B}$ tends to $B_{s}, d B / d H$ drops to minimum; implying from (3) that, $\mu$ tends to a minimum value $\mu_{\min }$.

Fig.2 shows the variation of the magnetic field density over one electrical rotation.

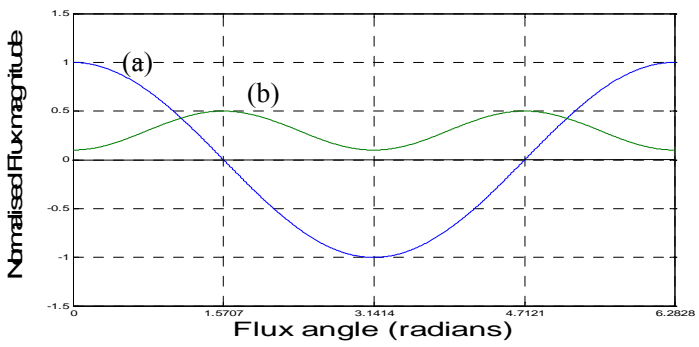

Fig.2. (a) Flux distribution over one electrical period; (b) permeability variation over the same electrical period note that the latter varies at twice the frequency of the fundamental flux distribution

The permeability therefore reaches a minimum twice per flux period thus giving rise to the $2 \mathrm{f}_{\mathrm{e}}$ modulation, where $f_{e}$ is electrical or flux frequency. This permeability modulation brings about an inductance modulation, which is in turn detected using the test pulse injection method described later on.

\section{SENSORLESS CONTROL OF AC MACHINES}

Sensorless control of AC machines has over the past years grown into one of the main areas of electric drive research. Various Sensorless speed and position control methods have been reported in literature
$[1,2,3,5,6$,$] . The sensorless control of AC machines can$ be categorised into two main classes: Model based or signal injection based.

Model based algorithms observe the motor speed or position through measured variables at the machine terminals and fundamental motor equations. Such methods however require correct commissioning of the motor parameters and are hence parameter dependent. Model based methods fail at zero and low speeds due to lack of signal and increased sensitivity to noise[2].

Signal injection based techniques inject a signal in addition to the fundamental machine excitation in the zero and low speed region to track a machine saliency. This makes such techniques parameter independent. The superimposed signal is of a high enough frequency to cause minimal disturbance to the fundamental operation of the drive [6]. Being of far greater frequency than the fundamental ,the hf signal goes through different paths in the machine than the fundamental signal, making it able to detect small machine anisotropies. The underlying idea is to exploit the anisotropy to identify the rotor/flux position information,(as was explained in the previous section the high frequency impedance paths, are modulated by either main flux or rotor bars; creating a position dependant impedance modulation).

Signal injection techniques are further subcategorized into two types: Carrier and pulse injection. This paper is concerned with the second type of signal injection.

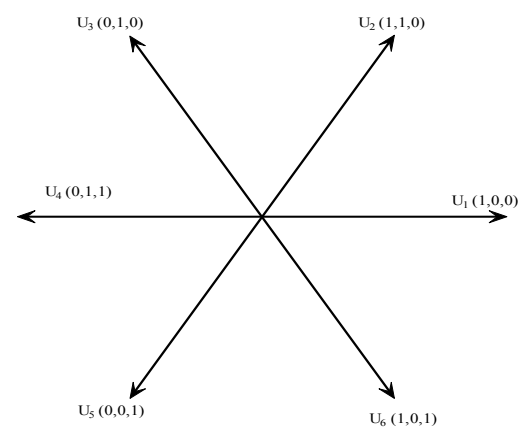

Fig.3. Voltage Test vectors

\section{TEST PULSE POSITION ESTIMATION METHOD}

In this method of position estimation, a voltage test pulse of short duration is applied followed immediately by its inverse [5](e.g. taking vector pair $\mathbf{u}_{1}-\mathbf{u}_{4}$ see state vector diagram fig.3). This is done so as not to disturb the fundamental voltage time area. Failure to do so will bring about a resultant fundamental flux density disturbance, which will effect the fundamental operation of the machine and might even be as severe as to cause torque pulsations.

At this point it is good to note that due to the high frequency nature of the injected pulse the leakage flux 
paths on the stator are excited, the observed effect is that of the stator leakage inductances being modulated by the main flux saturation. This variation is generally given in [1] by:

$$
\begin{aligned}
& l_{\sigma a}=l_{\sigma 0}+l_{\Delta} \cos (n \theta) \\
& l_{\sigma b}=l_{\sigma 0}+l_{\Delta} \cos \left(n\left(\theta-\frac{2 \pi}{3}\right)\right) \\
& l_{\sigma c}=l_{\sigma 0}+l_{\Delta} \cos \left(n\left(\theta-\frac{4 \pi}{3}\right)\right)
\end{aligned}
$$

where: $l_{\sigma 0}$ is the average inductance and $l_{\Delta}$ is the variation in magnitude due to the rotor anisotropy or saturation flux. ' $n$ ' assumes a value of 2 for saturation induced anisotropy or $n=N_{r} / p$ for rotor slotting were $N_{r}$ is the rotor slot number and $p$ the pole pairs. Considering the machine to be connected in star (the same method of analysis can be applied to a machine connected in a delta configuration), and that the applied vector is $\mathbf{u}_{1}(1,0,0)$, thus positive DC rail is applied to phase $\boldsymbol{a}$ whilst both $\boldsymbol{b}$ and $\boldsymbol{c}$ are shorted to the negative DC rail since $\mathrm{S} 1, \mathrm{~S} 4$ and $\mathrm{S} 5$ are $\mathrm{ON}$ as shown in Fig.4

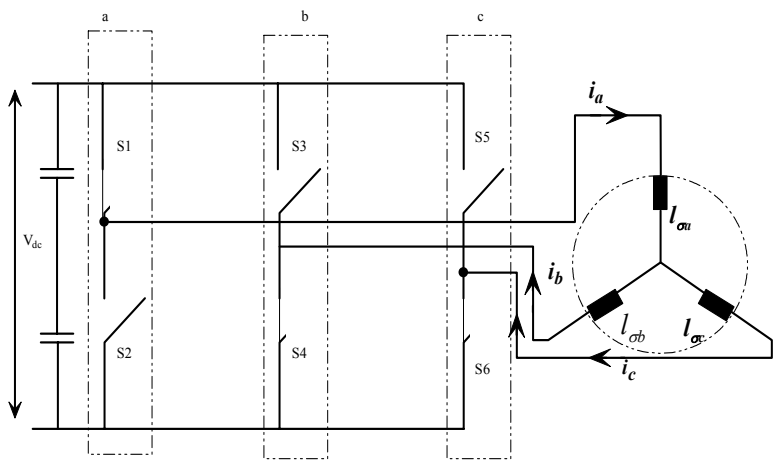

Fig.4. Schematic representation of the motor load showing the leakage inductances connected in star along with the inverter in switching state $\mathbf{u}_{1}$.

Assuming a relatively slow change in postion and using $v_{d c}=l(n \theta) \bullet d i / d t$, the derivative of the current impressed in each winding can be expressed in terms of both voltage vector applied and the position dependant leakage inductance by using equations (4). For the above case (vector $\mathbf{u}_{1}$ ):

$$
\begin{gathered}
\frac{d i_{a\left(u_{1}\right)}}{d t}=K\left(2-\frac{l_{\Delta}}{l_{\sigma 0}} \cos (n \theta)\right) \\
\frac{d i_{b\left(u_{1}\right)}}{d t}=-K\left(1+\frac{l_{\Delta}}{l_{\sigma 0}} \cos \left(n\left(\theta-\frac{4 \pi}{3}\right)\right)\right) \\
\frac{d i_{c}\left(u_{1}\right)}{d t}=-K\left(1+\frac{l_{\Delta}}{l_{\sigma 0}} \cos \left(n\left(\theta-\frac{2 \pi}{3}\right)\right)\right)
\end{gathered}
$$

were $\mathrm{K}$ is given by:

$$
K=\frac{v_{d c}}{3 l_{\sigma 0}\left(1-\left(\frac{l_{\Delta}}{2 l_{\sigma 0}}\right)^{2}\right)}
$$

It follows that a position signal can be extracted from measurement of the current derivatives. The sensor used for current derivative measurement is described later on in the paper.

Thus considering the equations derived in (5) for the active switching vector $\left(\mathbf{u}_{1}\right)$, the phase position components of the position vector can be expressed as:

$$
\begin{gathered}
p_{a\left(u_{1}\right)}=\frac{l_{\Delta}}{l_{\sigma 0}} \cos (n \theta)=-\frac{1}{K} \cdot \frac{d i_{a\left(u_{1}\right)}}{d t}+2 \\
p_{b\left(u_{1}\right)}=\frac{l_{\Delta}}{l_{\sigma 0}} \cos \left(n\left(\theta-\frac{2 \pi}{3}\right)\right)=-\frac{1}{K} \cdot \frac{d i_{c\left(u_{1}\right)}}{d t}-1 \\
p_{c\left(u_{1}\right)}=\frac{l_{\Delta}}{l_{\sigma 0}} \cos \left(n\left(\theta-\frac{4 \pi}{3}\right)\right)=-\frac{1}{K} \cdot \frac{d i_{b\left(u_{1}\right)}}{d t}-1
\end{gathered}
$$

This derivation may be repeated for all active switching states $\left(\mathbf{u}_{1}-\mathbf{u}_{6}\right)$ resulting in 18 position components (one per phase per vector/switching state). The position signals exhibit a dc offset as shown in (6). This effect is mitigated by using various combinations of the above derived position components to construct phase position components $\boldsymbol{p}_{\boldsymbol{a}}, \boldsymbol{p}_{\boldsymbol{b}}$ and $\boldsymbol{p}_{\boldsymbol{c}}$ as shown in (8).

Considering the phase position components $\boldsymbol{p}_{\boldsymbol{a}}, \boldsymbol{p}_{\boldsymbol{b}}$ and $\boldsymbol{p}_{\boldsymbol{c}}$ to be spatially displaced by $2 \pi / 3$ radians the resultant position tensor $\boldsymbol{p}_{\text {res }}$ for flux position estimation (substituting $\mathrm{n}=2$ in (6)) is given by:

$$
\begin{aligned}
& P_{\text {res }}=p \cdot e^{j n \theta}=C \cdot\left(\cos (2 \theta)+a \cos \left(2\left(\theta-\frac{2 \pi}{3}\right)\right)+a^{2} \cos \left(2\left(\theta-\frac{4 \pi}{3}\right)\right)\right) 7(\mathrm{a}) \\
& P_{r e s}=C \cdot\left(p_{a}+a p_{b}+a^{2} p_{c}\right)=p_{\alpha}+j p_{\beta} \quad 7(\mathrm{~b})
\end{aligned}
$$

where: $\underline{\boldsymbol{P}}_{\text {res }}$ is the resultant tensor, $\boldsymbol{p}_{\boldsymbol{a}} \boldsymbol{p}_{\boldsymbol{b}} \boldsymbol{p}_{\boldsymbol{c}}$ are the above mentioned phase position components, ' $a$ ' is a complex operator which denotes a phase shift of $2 \pi / 3$ radians and a multiplication of unit magnitude. $\boldsymbol{p}_{\boldsymbol{\alpha}}$ and $\boldsymbol{p}_{\boldsymbol{\beta}}$ are the quadrature components in the stationary frame $\boldsymbol{\alpha}-\boldsymbol{\beta}$.

In the present set up, a pair of voltage test vectors (these being $\mathbf{u}_{1}-\mathbf{u}_{4} ; \mathbf{u}_{3}-\mathbf{u}_{6} ; \mathbf{u}_{5}-\mathbf{u}_{2}$ ) are injected at the beginning of every fundamental PWM cycle as shown in Fig. 5 .

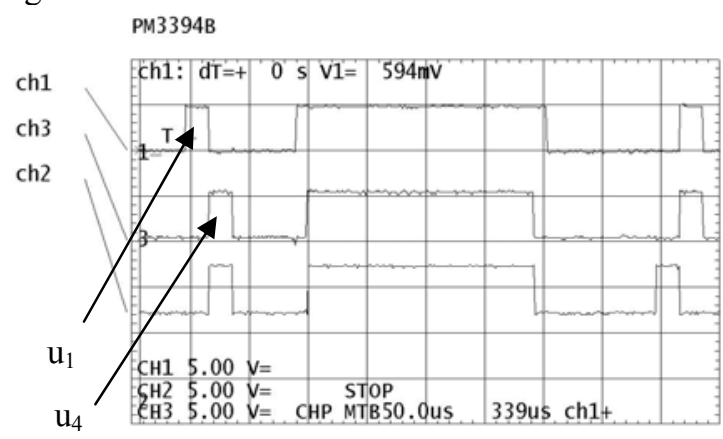

Fig.5. PWM output showing injection sequence $\mathbf{u}_{1}-\mathbf{u}_{4}$ at the beginning of the PWM interval.

Nine relevant position signals are extracted during vectors $\mathbf{u}_{1} ; \mathbf{u}_{3} ; \mathbf{u}_{5}$ resulting in phase position components $\mathrm{p}_{\mathrm{a}}, \mathrm{p}_{\mathrm{b}}, \mathrm{p}_{\mathrm{c}}$ given by:

$$
p_{a}=1 / 3 \cdot\left(p_{a\left(u_{1}\right)}+p_{a\left(u_{3}\right)}+p_{a\left(u_{5}\right)}\right)
$$




$$
\begin{aligned}
& p_{b}=1 / 3 .\left(p_{b\left(u_{1}\right)}+p_{b\left(u_{3}\right)}+p_{b\left(u_{5}\right)}\right) \\
& p_{c}=1 / 3 .\left(p_{c\left(u_{1}\right)}+p_{c\left(u_{3}\right)}+p_{c\left(u_{5}\right)}\right)
\end{aligned}
$$

$\mathbf{p}_{\text {res }}$ is then calculated by substituting the values obtained from (8) into (7b) thus obtaining the resultant quadrature components of the stationary frame $p_{\alpha} p_{\beta}$ in terms of the 9 position components shown in (6).The Estimated Flux angle $(\lambda)$, for the case of saturation saliency, is then obtained from :

$$
\lambda=\frac{1}{2} \tan ^{-1}\left(p_{\alpha} / p_{\beta}\right)
$$

\section{EXPERIMENTAL SETUP}

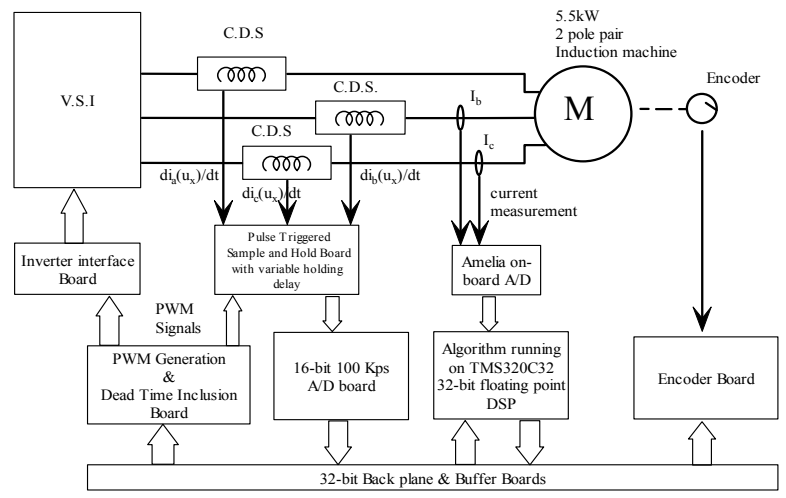

Fig.6. Experimental setup

Fig.6 above shows the basic layout of the experimental rig used. The star connected $5.5 \mathrm{~kW} 4-$ pole induction motor having semi-open rotor slots and skewed rotor; is controlled via the slip control algorithm running on a TMS320C32 DSP. A voltage signal proportional to the derivative of current passing to the motor is produced by the Current Derivative Sensors or CDS. A sample and hold circuit was built to differentiate between a test vector and the standard PWM signal and provides the required trigger for the sample and hold IC at $20 \mu \mathrm{s}$ after the test vector is triggered to start. This delay was introduced to allow for the current derivative signal to settle to a constant value. Data acquisition is carried out using the on board Amelia 16bit A/D for the monitoring the phase currents and another external faster $\mathrm{A} / \mathrm{D}$ is used for acquiring the relevant derivative signals.

The PWM switching frequency is set to $5 \mathrm{kHz}$ and has a settable dead time of $2.5 \mu \mathrm{s}$.

\section{CURRENT DERIVATIVE SENSORS}

The sensor topology investigated is based on the unity ratio coaxial air cored transformer suggested in [3]. The constructed prototype is shown in fig.7 below.

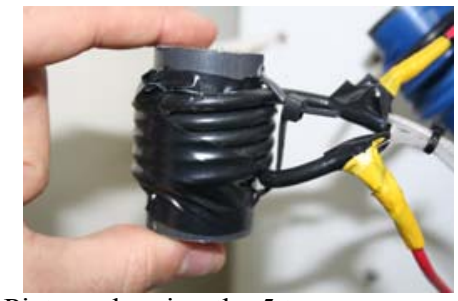

Fig.7. Picture showing the 5 turn coax sensor used

The sensor is made of five turns of $50 \Omega$ coaxial cable mounted on a plastic pipe. It is very easy to construct and the cost is negligible compared to the rest of the drive components. Two zener diodes placed in antiseries across the output of the coil secondary protect the instrumentation circuits to which the coil connects. This protection is required due to the presence of large voltage spikes which might develop during sharp current transients. The outer shield conducts the primary motor current, as it provides a larger cross sectional area, and the inner conductor is used as the secondary. The sensor gives a voltage signal proportional to the rate of change of flux setup and thus proportional to the rate of change of current. The output voltage is given by:

$$
V_{\text {dia }(u 1)}=\frac{-N_{2} N_{1} A_{c s a} \mu(B, H)}{l_{c}(N)} \bullet \frac{d i_{a(u 1)}}{d t}
$$

were $\mu(\mathrm{B}, \mathrm{H})$ is the effective magnetic permeability of the core material (variable with magnetic excitation)and can assume a value of $\mu_{0}=4 \pi \times 10^{-7}$ if the core is made out of paramagnetic materials such as air in the case of the coax sensor; $\mathrm{N}_{1}$ is the amount of turns making up the primary in this case $\mathrm{N}_{1}=5, \mathrm{~N}_{2}$ are the secondary turns in this case $\mathrm{N}_{2}=5 . A_{c s a}$ is the surface area of the flux linkage path (cross-sectional area of the solenoid if linear or of the toroidal core in the case of a toroid), $l(\mathrm{~N})$ is the coil length and finally $d i_{\mathrm{a}(\mathrm{u} 1)} / d t$ is the derivative of the 'a' phase current when the voltage test vector $\boldsymbol{u}_{1}$ is applied. The settling time, amplitude, and signal to noise ratio of the outputted signal is investigated.

\section{RESULTS}

The CDS shown in Fig.7 was initially tested for its response, when a current ramp having a di/dt of $1.4 \mathrm{~A}$ per $20 \mu$ s is applied shown in Fig.8. (This approximates to a $d i / d t$ of $70000 \mathrm{Amps}$ per second). In order to provide perfectly smooth current slope as a correct bases of assessment only the rising edge of the current waveform is considered. The main criterion of performance assessment is the settling time required for a stable derivative value to be sampled. The sensor response is shown in Fig. 9 where it can be seen that it gives a signal of magnitude $45 \mathrm{mV}$ with a settling time of $10.5 \mu \mathrm{s}$. 
The current derivative sensors were implemented on the experimental set-up shown in fig 6. The experimental results of the final alpha-beta components of the flux position signals along with their frequency spectra and resulting estimated flux angle are shown in Figs 10 to 13 .

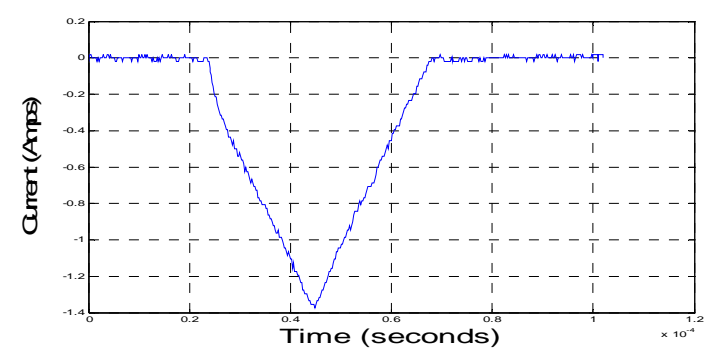

Fig.8. Waveform showing the current ramp applied

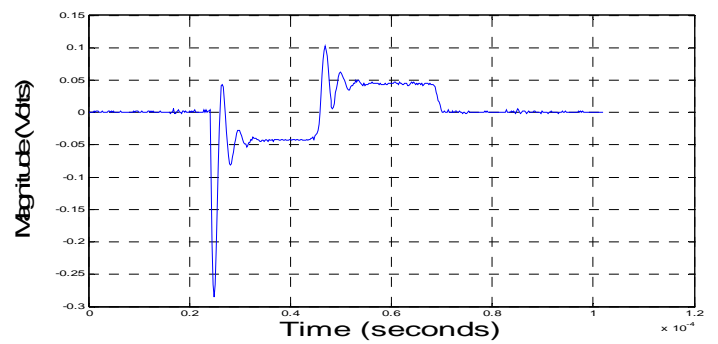

Fig.9. response of Coax current derivative sensor A5T, having a turns ratio of 5:5. Signal magnitude at $45 \mathrm{mV}$ with a settling time of $10.5 \mu \mathrm{s}$
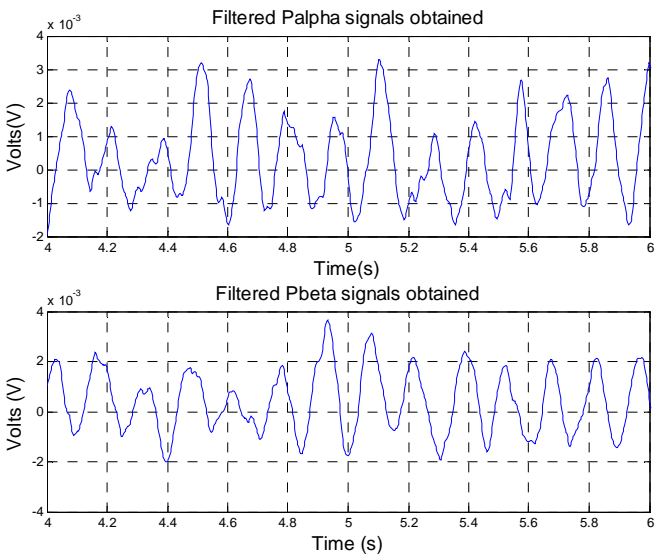

Fig. 10 Filtered time domain signals for $\mathbf{p} \alpha$ and $\mathbf{p} \beta$

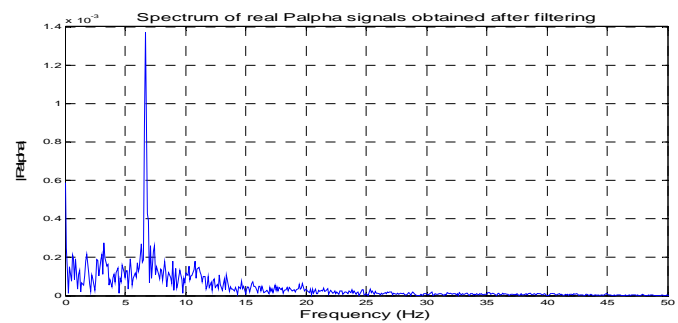

Fig.11. Frequency spectrum of Alpha component $\mathbf{p}_{\alpha}$ of the resultant flux position

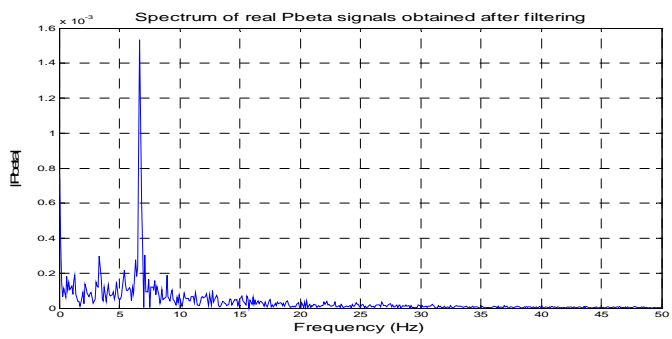

Fig.12. Frequency spectrum of Beta component $\mathbf{p}_{\beta}$ of the resultant flux position

The extracted quadrature signals are filtered using a $1^{\text {st }}$ order low pass Butterworth filter with cutoff frequency set at $20 \mathrm{~Hz}$. The time domain signals are shown in Fig.10. Figs. 11 and 12 show the spectra of the respective quadrature components of the position signal in the stationary frame $\alpha-\beta$, where the main saturation signal at $2 f_{e}$ can be seen. Finally Fig. 13 below shows the estimated flux angle reconstructed from (9).

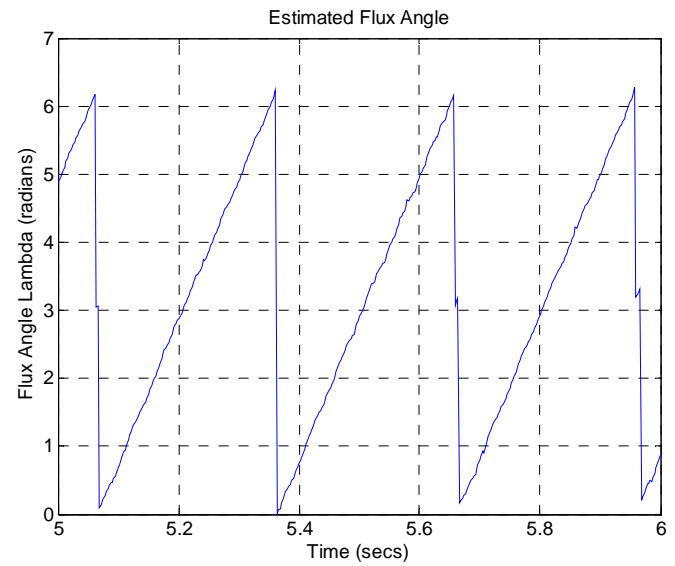

Fig.13. Estimated Flux angle $(\lambda)$

\section{CONCLUSION}

This paper has shown the use of inexpensive current derivative sensors for measuring the line current derivatives during the injection of voltage test vectors. Saturation saliency modulates the current derivatives and allows tracking of the flux position in induction machines. This can be used for sensorless torque control in the zero and low speed region.

\section{REFERENCES}

[1] J.Holtz and J. Juliet "sensorless Acquisition of the rotor Position Angle for Induction Motors with arbitrary stator windings" 0-7803-8487-3/04 IEEE2004

[2]C.Caruana et al. "Sensorless flux position estimation at low and zero frequency by measuring Zero-Sequence Current in Delta Connected Cage Induction Machines" IEEE Transactions on Industry Applications Vol.41, Issue 2, March-April 2005 Page(s):609 - 617 
[3] J.Holtz, "Sensorless position control of induction motorsan emerging technology" IEEE Transactions on Industrial

Electronics no6 vol45 pp840-852 Dec 1998

[4] Wolbank et al "Closed-Loop Compensating Sensors

Versus new Current Derivative Sensors"IEEE Transactions

on Instrumentation and Measurement Vol53 No.4 Aug 2004.

[5] M. Schrödl, "Sensorless Control of AC Machines at Low Speed and Standstill Based on the INFORM Method," IEEE

IAS Annual Meeting, San Diego, USA, Oct. 1996, Vol. 1 pp. 270-277.

[6] N.Teske, C.Spiteri Staines, J.Cilia, G.M.Asher,

"Sensorless Position and Speed Estimation in Induction Machines", EPE-PEMC 2002, Croatia. 